\title{
Indications, advantages, disadvantages and effectiveness of Invisalign aligners
}

\author{
Mohamed A. Abdelfattah Hosny ${ }^{1 *}$, Fahad S. Alasmari ${ }^{2}$, Nada M. Alsaidi ${ }^{3}$, Hanin M. Alsharif ${ }^{4}$, \\ Sewar A. Alshareef ${ }^{5}$, Nora F. Aldwyyan ${ }^{3}$, Rahaf Y. Alahmadi ${ }^{3}$, Ruba A. Almutairi ${ }^{6}$, \\ Bashayer M. Almutairi ${ }^{7}$, Ghufran S. Alhemaidi ${ }^{3}$ Ebtihal T. Alahdal $^{8}$
}

\author{
${ }^{1}$ Department of Orthodontics, Andalusia Hospitals, Jeddah, Saudi Arabia \\ ${ }^{2}$ Primary Health Care, Ministry of Health, Abha, Saudi Arabia \\ ${ }^{3}$ General Dentist, Ministry of Health, Madinah, Saudi Arabia \\ ${ }^{4}$ General Dentist, Dental Specialist Center, Tabuk, Saudi Arabia \\ ${ }^{5}$ General Dentist, Ministry of Health, Riyadh, Saudi Arabia \\ ${ }^{6}$ General Dentist, University of Jordan, Amman, Jordan \\ ${ }^{7}$ General Dentist, Dr Abdulaziz Dental Clinics, Riyadh, Saudi Arabia \\ ${ }^{8}$ Department of Dentistry, King Abdulaziz University, Jeddah, Saudi Arabia
}

Received: 06 September 2021

Accepted: 09 September 2021

\section{*Correspondence:}

Dr. Mohamed A. Abdelfattah Hosny,

E-mail: mohakmal317@gmail.com

Copyright: () the author(s), publisher and licensee Medip Academy. This is an open-access article distributed under the terms of the Creative Commons Attribution Non-Commercial License, which permits unrestricted non-commercial use, distribution, and reproduction in any medium, provided the original work is properly cited.

\begin{abstract}
As a result of the increasing demands to enhance esthetics among the different community populations, many efforts have focused on innovating other substitutes to increase patients' satisfaction and to obtain better clinical outcomes. Among the variously proposed candidates, clear aligners are now commonly reported and used within clinical settings. Additionally, technological advances are also significant in this field. In this literature review, we aim to discuss the indications, advantages, disadvantages and the effectiveness of Invisalign braces. Typically, the ideal patients that are indicated to receive Invisalign systems are adult patients with either incisor flaring, spacing, crowding, infra- or suprapositions. Although time-consuming, Invisalign systems are reported with reduced adverse events and toxic reactions with the gingival tissue, minimizing the risk of developing periodontal diseases. However, Although Invisalign modalities are associated with fewer adverse events and reduced gingival inflammation and plaque formation. Previous studies have demonstrated that the outcomes might be similar when patients with fixed appliances are educated about properly conducting oral hygienes. Besides, improved healing of the periodontal ligament was also reported with the modality. Therefore, better clinical outcomes are expected with the modality. Furthermore, studies have also demonstrated similar effectiveness between Invisalign and fixed appliances while the most accurately obtained tooth movements with Invisalign might include retrusion, rotations, fan-type expansions and protrusions. Further investigations are urgently needed for further validation of the current evidence.
\end{abstract}

Keywords: Orthodontics, Invisalign, Aligners, Fixed appliances, Esthetics

\section{INTRODUCTION}

As a result of the increasing demands to enhance esthetics among the different community populations, many efforts have focused on innovating other substitutes to increase patients' satisfaction and to obtain better clinical outcomes. ${ }^{1,2}$ Among the variously proposed candidates, clear aligners are now commonly reported and used within clinical settings. Additionally, technological advances are also significant in this field. ${ }^{1}$ Many merits have been 
reported with clear aligners, including Invisalign, as easy oral hygiene, enhanced esthetics and patients' satisfaction, and reduced number of visits to the clinics and emergency department. ${ }^{3,4}$ On the other hand, many limitations to these modalities were also reported in the literature, as the high cost, limited uses, being ineffective with some malocclusions, and their significant dependence on patients' cooperation and compliance. ${ }^{1,5-8}$ In this literature review, we aim to discuss the indications, advantages, disadvantages and the effectiveness of Invisalign braces.

\section{DISCUSSION}

\section{Advantages and disadvantages}

Many advantages and disadvantages were reported with using Invisalign braces among studies in the literature as compared to the fixed orthodontic appliances (Figure 1). Among the reported advantages, studies have demonstrated that Invisalign braces have minimal or no adverse events on the gingival tissues. ${ }^{9}$ The appliances were reported to have a positive impact on the relevant teeth, with reduced chances of periodontal diseases and hypomineralization because they are not usually associated with interfering with the oral hygiene measures. ${ }^{10,11}$ This was furtherly indicated in an investigation by Turatti et al. ${ }^{12} \mathrm{He}$ reported that Invisalign was associated with minimal adverse events in a patient who was already suffering from periodontal disease and intruded incisors. Furthermore, it was previously noted that the Invisalign braces do not usually interfere with the movement of the tongue, which is better than the lingual fixed appliances. ${ }^{13,14}$ It was also reported that the Invisalign braces lead to the eradication of the labial fixed appliances appearance. Accordingly, estimates show that the Invisalign compounds are the most widely chosen and accepted modalities among women suffering from speech and esthetic problems that are usually associated with the fixed appliances. It was also previously theoretically reported that Invisalign braces are usually associated with less discomfort because the braces are periodically removed which gives an adequate chance for the periodontal ligament to heal during drinking, eating, and cleaning of the appliance and teeth. ${ }^{15}$ This was furtherly indicated in a survey-based investigation by Nedwed and Miethke that included 54 patients with Invisalign braces to investigate some parameters related to the effectiveness of the appliance as development and duration of pain, adaptation time, symptoms related to the tempomandibular joint, the presence of mucosal and lingual irritation, impairment of speech, and patients' satisfaction and reporting of the relevant information since the modality was installed. ${ }^{16}$ The authors reported favorable outcomes, and patients generally showed high acceptance levels of the effectiveness of the modality. The adaptation period for Invisalign patients has been estimated to be one week only and most patients reported they were not bothered about it. Mild pain for 2-3 days or minimal mucosal irritations might also be experienced. No significant speech impairment was noticed with no tempo- mandibular joint affection, although some patients reported the presence of associated clicking. However, it was presented before the therapy was inaugurated. Another prospective investigation by Miller et al included 60 patients that were treated with Invisalign and fixed appliances to assess the quality of life following the first week of the treatment procedure. ${ }^{4}$ The authors reported that the adverse events and pain occurrence were significantly more frequent in patients within the Invisalign group than the fixed appliances one. Nevertheless, it should be noted that the results were based on patients' reports according to daily diaries based on their experiences. Invisalign appliances are also associated with a reduced risk of resorption, which is potentially due to the associated low forces as teeth are not removed by more than $0.2 \mathrm{~mm}$ when installing the modality. This was supported by the results of a previous randomized controlled trial by Barbagallo et al. ${ }^{17}$ Besides, a previous investigation by Brezniak and Wasserstein also reported that severe resorption was significantly associated with the installation of an Invisalign system in their patient. ${ }^{18}$ Furthermore, additional studies have also reported the efficacy of the recent techniques in reporting the movement outcomes with Invisalign systems. ${ }^{19,20}$

Lingual constrictions and extrusions were the most and the least reliable movement with Invisalign systems, respectively, as reported by Kravitz et al which also showed that Invisalign systems were only able to accomplish a total of $41 \%$ of the programmed teeth movements. ${ }^{21}$ Finally, using Invisalign has been associated with fewer emergencies, decreased clinical visits, and reduced armamentarium frequencies. In addition, they are being successfully used in patients with bonding challenges, as in cases of multiple artificial tooth surfaces. It should be noted that the effectiveness of the Invisalign systems is limited by the difficulty to change the system once it has been fabricated and set to be installed. ${ }^{22}$ Using additional aligners or fixed appliances might even be needed in cases of non-satisfactory results. Although esthetics of the Invisalign systems are widely acceptable by patients, this advantage might be limited by the long time needed by the modality that is similar to other appliances. $^{23}$ To overcome this issue, a previous investigation by Owen et al reported that using Invisalign together with accelerated osteogenic orthodontics to fasten the process and enhance patient satisfaction. ${ }^{23}$ Besides, as polyurethane is abundantly present in Invisalign modalities, issues related to biocompatibility might be present because the substance can be impacted by moisture, heat, and increased contact with enzymes. Accordingly, some functional and morphological observations might be associated as a result of these events. $^{24,25}$

Other disadvantage is the nature of dental caries formations. It is known that saliva carry high numbers of bacteria. When the numbers of salivary bacteria exceeds $105 \mathrm{CFU} / \mathrm{ml}$, a high risk of developing bacteria develops. This can be attributed to the fact that any foreign body in 
the oral cavity can be responsible for producing more saliva with aligners being the culpirt in such cases. ${ }^{26}$

\begin{tabular}{|c|c|}
\hline Advantages of Invisalign & Disadvantages of Invisalign ${ }^{*}$ \\
\hline Ideal aesthetics & $\begin{array}{l}\text { Limited control over root } \\
\text { movement }\end{array}$ \\
\hline Ease of use for the patient & $\begin{array}{l}\text { Limited intermaxillary correction } \\
\text { (severe skeletal discrepancies } \\
\text { cannot be contemplated with } \\
\text { Invisalign } \\
\end{array}$ \\
\hline Comfort of wear & $\begin{array}{l}\text { Lack of operator control (the } \\
\text { clinician has no ability to alter the } \\
\text { appliance during the course of } \\
\text { treatment) }\end{array}$ \\
\hline $\begin{array}{l}\text { Simplicity of care and } \\
\text { better oral hygiene }\end{array}$ & $\begin{array}{l}\text { Additional time and/or } \\
\text { documentation required if changes } \\
\text { have to be made once the } \\
\text { treatment has started }\end{array}$ \\
\hline $\begin{array}{l}\text { Potential metal allergy reactions } \\
\text { associated with conventional } \\
\text { fixed appliances are avoided }\end{array}$ & $\begin{array}{c}\text { Slight intrusion }(0.25-0.5 \mathrm{~mm}) \\
\text { of the posterior teeth may occur } \\
\text { (it is corrected during the retention } \\
\text { period) }\end{array}$ \\
\hline \multicolumn{2}{|l|}{$\begin{array}{l}\text { Elimination of the difficulty } \\
\text { of bonding fixed appliances }\end{array}$} \\
\hline \multicolumn{2}{|l|}{$\begin{array}{l}\text { Evaluation of treatment options in } \\
\text { detail before beginning treatment }\end{array}$} \\
\hline $\begin{array}{l}\text { The virtual treatment model can } \\
\text { serve as a motivating tool for the } \\
\text { patient }\end{array}$ & \\
\hline
\end{tabular}

Figure 1: Advantages and disadvantages of Invisalign. ${ }^{5}$

\section{Indications and effectiveness}

Typically, the ideal patients that are indicated to receive Invisalign systems are adult patients with either incisor flaring, spacing, crowding, or infra- or supra-positions. ${ }^{4,27}$ A previous investigation by Joffe et al summarized the indications when Invisalign systems should be considered based on the patients' symptoms. ${ }^{28}$ In addition, the situations should be considered when Invisalign is usually of limited effectiveness. The reported symptoms by which the patient is an ideal candidate to receive management by Invisalign systems to include: spacing is $\leq 5 \mathrm{~mm}$, crowding is $\leq 5 \mathrm{~mm}$, having constricted arches where correction is needed to avoid the development of unnecessary buccal tipping, and the presence of deep bite class II division 2 where management is done by protrusions and intrusions of the incisors. On the other hand, symptoms that do not favor the use of the Invisalign modalities include: the presence of anteroposterior skeletal discrepancies, crowding is $>5 \mathrm{~mm}$, teeth extrusion, posterior and anterior open bites, severe rotations $>20^{\circ}$, centric occlusion or relation discrepancies, severe oligodontia or hypodontia, and the presence of uprighted severely tipped teeth $>45^{\circ}$. This list was furtherly evaluated and validated by other investigations. For instance, Clements et al reported that the last improvements were noticed with the buccal occlusions while the earliest were noticed with the anterior alignment. $^{9}$ Besides, incisors closing was more significantly enhanced as compared to the mandibular and maxillary extraction sites, as indicated by the authors. In the same context, a previous study by Bollen et al also showed that patients with no extractions, two-week activation regimens, and reduced baseline peer assessment rating scores were associated with enhanced outcomes following single management by an aligner modality. ${ }^{29}$ Furthermore, Joffe also indicated that as a result that some issues as malocclusions and other clinical manifestations might not be adequately treated with Invisalign systems from the first management approach, combining the modality with other approaches is recommended in such situations to enhance the outcomes. ${ }^{28}$ In this context, a previous study by Giancotti showed that bonding of the labial and lingual buttons with elastics was effective in closing the space that resulted from a missing mandibular first molar. ${ }^{30}$ Besides, it was previously demonstrated that Invisalign systems can be effectively used to enhance the outcomes related to severe skeletal malocclusions within orthognathic surgeries. ${ }^{31,32}$ Moreover, it was furtherly reported that full-fixed or fixed appliances were used in these patients before and after performing the surgeries. In combination with lingual brackets and mini-screws, Invisalign systems were also effectively reported. ${ }^{33}$ Another treatment protocol was also generated by Boyd et al and was also validated and adopted by align technology to be generalized within the clinical settings to achieve better outcomes. ${ }^{34}$

Among the various studies in the literature, evidence is abundant regarding the effectiveness of using Invisalign systems as compared to other treatment modalities. In general, it has been reported that the management outcomes with the Invisalign systems are inferior to the fixed appliances. ${ }^{35}$ It was demonstrated that issues regarding the optimization of the occlusal defects and contacts, in addition to managing the discrepancies of the anteroposterior skeletal cannot be fixed using the Invisalign systems. A previous investigation also concluded that fixed appliances are associated with higher treatment stability rates as compared to the Invisalign systems. ${ }^{36}$ However, the design of the investigation should be considered when interpreting these findings, as it was a retrospective cohort study. Besides, this conclusion was not furtherly validated by a following systematic review. ${ }^{37}$ Lower canines have been reported to be the most difficult to be controlled using the Invisalign systems. A previous investigation by Weihong et al compared the outcomes and effectiveness of both Invisalign and fixed appliances in moderate and mild cases that were managed with premolar extractions. ${ }^{38}$ Favorable outcomes were obtained by both modalities. However, previous studies have demonstrated that adequate experience and knowledge are two important factors to obtain acceptable outcomes in such situations. ${ }^{39,40}$ The most accurately obtained tooth movements might also include retrusion, rotations, fantype expansions, and protrusions. ${ }^{41}$ Another investigation by Djeu et al that compared the effectiveness of Invisalign with fixed braces found that posterior torque and occlusal contacts were significantly more common with Invisalign 
than the fixed appliances in managing the musculoskeletal discrepancies, while both modalities were effectively equal in terms of root paralleling, alignment of the marginal ridges and space closure. ${ }^{35}$ Furthermore, studies in the literature have also evaluated the effect of Invisalign on periodontal outcomes, and many have reported that the system has a positive impact. ${ }^{42,43}$ Although we previously reported that Invisalign modalities are associated with fewer adverse events and reduced gingival inflammation and plaque formation, previous studies have demonstrated that the outcomes might be similar when patients with fixed appliances are educated about properly conducting oral hygienes. ${ }^{42}$ Besides, it should be noted that white spot lesions might be observed in cases when Invisalign systems were applied as a result of the reduced plaque accumulation in these areas. ${ }^{44}$

\section{CONCLUSION}

Typically, the ideal patients that are indicated to receive Invisalign systems are adult patients with either incisor flaring, spacing, crowding, or infra- or supra-positions. Although time-consuming, Invisalign systems are reported with reduced adverse events and toxic reactions with the gingival tissue, minimizing the risk of developing periodontal diseases. Besides, improved healing of the periodontal ligament was also reported with the modality, and therefore, better clinical outcomes are expected with the modality. Further investigations are urgently needed for further validation of the current evidence.

\section{Funding: No funding sources \\ Conflict of interest: None declared \\ Ethical approval: Not required}

\section{REFERENCES}

1. Weir T. Clear aligners in orthodontic treatment. Aust Dent J. 2017;62(1):58-62.

2. Malik $\mathrm{OH}$, McMullin A, Waring DT. Invisible orthodontics part 1: invisalign. Dent Update. 2013;40(3):203-4.

3. Fujiyama K, Honjo T, Suzuki M, Matsuoka S, Deguchi T. Analysis of pain level in cases treated with Invisalign aligner: comparison with fixed edgewise appliance therapy. Progress in orthodontics. 2014;15(1):64.

4. Miller KB, McGorray SP, Womack R, Quintero JC, Perelmuter M, Gibson J, et al. A comparison of treatment impacts between Invisalign aligner and fixed appliance therapy during the first week of treatment. Am J Orthod Dentofacial Orthop. 2007;131(3):302.

5. Melkos AB. Advances in digital technology and orthodontics: a reference to the Invisalign method. Med Sci Monit. 2005;11(5):I39-42.

6. Wheeler TT. Orthodontic clear aligner treatment. Paper presented at: Seminars in Orthodontics. 2017.

7. Proffit WR, Fields HW, Sarver DM. Contemporary orthodontics. Elsevier Health Sciences. 2006.
8. Nahoum HI. Forces and moments generated by removable thermoplastic aligners. Am J Orthod Dentofacial Orthop. 2014;146(5):545-6.

9. Clements KM, Bollen AM, Huang G, King G, Hujoel $\mathrm{P}, \mathrm{Ma}$ T. Activation time and material stiffness of sequential removable orthodontic appliances. Part 2: Dental improvements. Am J Orthod Dentofacial Orthop. 2003;124(5):502-8.

10. Miethke RR, Vogt S. A comparison of the periodontal health of patients during treatment with the Invisalign system and with fixed orthodontic appliances. J Orofacial Orthop. 2005;66(3):219-29.

11. Miethke RR, Brauner K. A Comparison of the periodontal health of patients during treatment with the Invisalign system and with fixed lingual appliances. J Orofacial Orthop. 2007;68(3):223-31.

12. Turatti G, Womack R, Bracco P. Incisor intrusion with Invisalign treatment of an adult periodontal patient. J Clin Orthod. 2006;40(3):171-4.

13. Giancotti A, Mampieri G, Greco M. Correction of deep bite in adults using the Invisalign system. J Clin Orthod. 2008;42(12):719-26.

14. Meier B, Wiemer KB, Miethke RR. Invisalign-patient profiling. Analysis of a prospective survey. $\mathbf{J}$ Orofacial Orthop. 2003;64(5):352-8.

15. Rosvall MD, Fields HW, Ziuchkovski J, Rosenstiel SF, Johnston WM. Attractiveness, acceptability, and value of orthodontic appliances. Am J Orthod Dentofacial Orthop. 2009;135(3):276.

16. Nedwed V, Miethke RR. Motivation, acceptance and problems of invisalign patients. J Orofacial Orthop. 2005;66(2):162-73.

17. Barbagallo LJ, Jones AS, Petocz P, Darendeliler MA. Physical properties of root cementum: Part 10 . Comparison of the effects of invisible removable thermoplastic appliances with light and heavy orthodontic forces on premolar cementum. A microcomputed-tomography study. Am J Orthod Dentofacial Orthop. 2008;133(2):218-27.

18. Brezniak N, Wasserstein A. Root resorption following treatment with aligners. Angle Orthod. 2008;78(6):1119-24.

19. Miller RJ, Kuo E, Choi W. Validation of Align Technology's Treat III digital model superimposition tool and its case application. Orthod Craniofacial Res. 2003;6(1):143-9.

20. Beers AC, Choi W, Pavlovskaia E. Computerassisted treatment planning and analysis. Orthod Craniofacial Res. 2003;6(1):117-125.

21. Kravitz ND, Kusnoto B, BeGole E, Obrez A, Agran B. How well does Invisalign work? A prospective clinical study evaluating the efficacy of tooth movement with Invisalign. Am J Orthod Dentofacial Orthop. 2009;135(1):27-35.

22. Phan X, Ling PH. Clinical limitations of Invisalign. J Canad Dent Assoc. 2007;73(3):263-6.

23. Owen AH. Accelerated Invisalign treatment. J Clin Orthod. 2001;35(6):381-5.

24. Schuster S, Eliades G, Zinelis S, Eliades T, Bradley TG. Structural conformation and leaching from in 
vitro aged and retrieved Invisalign appliances. Am J Orthod Dentofacial Orthop. 2004;126(6):725-8.

25. Kwon JS, Lee YK, Lim BS, Lim YK. Force delivery properties of thermoplastic orthodontic materials. Am J Orthod Dentofacial Orthop. 2008;133(2):228-34.

26. Mummolo S, Nota A, Albani F. Salivary levels of Streptococcus mutans and Lactobacilli and other salivary indices in patients wearing clear aligners versus fixed orthodontic appliances: An observational study. Plos One. 2020;15(4):e0228798.

27. Vlaskalic V, Boyd R. Orthodontic treatment of a mildly crowded malocclusion using the Invisalign System. Aust Orthod J. 2001;17(1):41-6.

28. Joffe L. Invisalign: early experiences. J Orthod. 2003;30(4):348-52.

29. Bollen AM, Huang G, King G, Hujoel P, Ma T. Activation time and material stiffness of sequential removable orthodontic appliances. Part 1: Ability to complete treatment. Am J Orthod Dentofacial Orthop. 2003;124(5):496-501.

30. Giancotti A, Ronchin M. Pre-restorative treatment with the Invisalign system. $\mathbf{J}$ Clin Orthod. 2006;40(11):679-82.

31. Boyd RL. Surgical-orthodontic treatment of two skeletal Class III patients with Invisalign and fixed appliances. J Clin Orthod. 2005;39(4):245-58.

32. Womack WR, Day RH. Surgical-orthodontic treatment using the Invisalign system. J Clin Orthod. 2008;42(4):237-45.

33. Choi NC, Park YC, Jo YM, Lee KJ. Combined use of miniscrews and clear appliances for the treatment of bialveolar protrusion without conventional brackets. Am J Orthod Dentofacial Orthop. 2009;135(5):671-81.

34. Boyd RL. Complex orthodontic treatment using a new protocol for the Invisalign appliance. J Clin Orthod. 2007;41(9):525-47.

35. Djeu G, Shelton C, Maganzini A. Outcome assessment of Invisalign and traditional orthodontic treatment compared with the American Board of Orthodontics objective grading system. Am J Orthod Dentofacial Orthop. 2005;128(3):292-8.
36. Kuncio D, Maganzini A, Shelton C, Freeman K. Invisalign and traditional orthodontic treatment postretention outcomes compared using the American Board of Orthodontics objective grading system. Angle Orthod. 2007;77(5):864-9.

37. Lagravère MO, Flores-Mir C. The treatment effects of Invisalign orthodontic aligners: a systematic review. J Am Dent Assoc. 2005;136(12):1724-9.

38. Li W, Wang S, Zhang Y. The effectiveness of the Invisalign appliance in extraction cases using the the ABO model grading system: a multicenter randomized controlled trial. Int $\mathrm{J}$ Clin Exp Med. 2015;8(5):8276-82.

39. Hönn M, Göz G. A premolar extraction case using the Invisalign system. $\mathrm{J}$ Orofacial Orthop. 2006;67(5):385-94.

40. Rossini G, Parrini S, Castroflorio T, Deregibus A, Debernardi CL. Efficacy of clear aligners in controlling orthodontic tooth movement: a systematic review. Angle Orthod. 2015;85(5):881-9.

41. Johnson K. Doctoral Dissertations. J Real Estate Literature. 2005;13(3):357-62.

42. Han JY. A comparative study of combined periodontal and orthodontic treatment with fixed appliances and clear aligners in patients with periodontitis. J Periodont Implant Sci. 2015;45(6):193-204.

43. Abbate GM, Caria MP, Montanari P. Periodontal health in teenagers treated with removable aligners and fixed orthodontic appliances. J J Orofacial Orthop. 2015;76(3):240-50.

44. Azeem M, Hamid WU. Incidence of white spot lesions during orthodontic clear aligner therapy. J World Federation of Orthodontists. 2017;6(3):127-30.

Cite this article as: Hosny MAA, Alasmari FS, Alsaidi NM, Alsharif HM, Alshareef SA, Aldwyyan $\mathrm{NF}$, et al. Indications, advantages, disadvantages and effectiveness of Invisalign aligners. Int J Community Med Public Health 2021;8:5064-8. 